\title{
A new DSI-SLM method for PAPR reduction in OFDM systems
}

\begin{abstract}
High PAPR is the main drawback of OFDM systems. DSI method and SLM method are two of the most promising techniques to reduce PAPR.
\end{abstract}

